\title{
RESISTÊNCIA DE AMENDOIM - BRAVO AOS HERBICIDAS INIBIDORES DA ENZIMA ALS ${ }^{1}$
}

DIONISIO L. P. GAZZIERO ${ }^{2}$, ALEXANDRE M. BRIGHENTI ${ }^{2}$, CLEBER D. G. MACIEL ${ }^{3}$, PEDRO J. CHRISTOFOLLETI ${ }^{4}$, FERNANDO S. ADEGAS ${ }^{5}$ e ELEMAR VOLL ${ }^{2}$

\section{RESUMO}

Uma das práticas agrícolas mais eficientes para o controle de plantas daninhas na agricultura é o uso de herbicidas seletivos. Todavia, a aplicação de um mesmo herbicida, ou de herbicidas com o mesmo mecanismo de ação, durante anos consecutivos, na mesma área, pode resultar na seleção de biótipos de plantas daninhas resistentes a esses herbicidas. Com o objetivo de confirmar a resistência de um biótipo (E-298) da planta daninha amendoim-bravo (Euphorbia heterophylla) aos herbicidas inibidores da enzima ALS, encontrado em área de cultivo de soja, no município de Cafelândia, PR, foi conduzido um experimento em área pertencente à Embrapa Soja, Londrina, PR. As plantas provenientes da população com suspeita de resistência foram tratadas com diversos herbicidas e doses, e comparadas com plantas de uma população suscetível de amendoim-bravo. Os tratamentos foram estabelecidos considerando as doses recomendadas dos herbicidas, metade delas e, duas, quatro e oito vezes superiores a recomendada. Os produtos e as doses aplicadas foram cloransulan nas doses 0,$0 ; 0,015 ; 0,03 ; 0,06$; 0,$12 ; 0,24 \mathrm{~kg}$ i.a./ha, mais o espalhante adesivo Agral $0,2 \% \mathrm{v} / \mathrm{v}$, imazetaphapyr nas doses 0,0 ;
0,$05 ; 0,1 ; 0,2 ; 0,4$ e $0,8 \mathrm{~kg}$ i.a./ha, imazaquin nas doses 0,$0 ; 0,075 ; 0,15 ; 0,3 ; 0,6 ; 1,2 \mathrm{~kg}$ i.a./ha, mais o espalhante adesivo Assist $0,3 \% \mathrm{v} / \mathrm{v}$, sulfentrazone nas doses 0,$0 ; 0,3 ; 0,6 ; 1,2 ; 2,4$; $4,8 \mathrm{~kg}$ i.a./ha, mais o espalhante adesivo Assist $0,3 \% \mathrm{v} / \mathrm{v}$, e lactofen nas doses 0,$0 ; 0,082 ; 0,165$; 0,$33 ; 0,664 ; 1,329 \mathrm{~kg}$ i.a./ha. O biótipo resistente apresentou diferentes níveis de resistência cruzada entre os herbicidas cloransulan, imazethapyr e o imazaquin, e as relações médias de $\mathrm{GR}_{50}$ obtidas foram $>6,85 ; 11,90$ e $>7,18$, respectivamente. As curvas de dose-resposta do biótipo resistente apresentaram valores inferiores ao biótipo suscetível, em todas as doses estudadas para os herbicidas cloransulan, imazethapyr e imazaquin. Para os herbicidas sulfentrazone e lactofen, que apresentam mecanismo de ação diferente da inibição da enzima ALS, ocorreu alto índice de controle de ambos os biótipos de amendoim-bravo avaliados, principalmente, nas maiores doses, provando a não existência de resistência múltipla nesse biótipo de amendoim-bravo estudado.

Palavras chave: resistência cruzada, resistência múltipla, planta daninha, Euphorbia heterophylla.

\section{ABSTRACT}

\section{Resistance of the weed wild poinsettia to ALS inhibitor herbicides}

One of the most efficient methods of weed control in agriculture is the use of selective herbicides. However, repetitive use of the same herbicide or herbicides having the same mechanism of action, has led to the appearance of weed biotypes which have developed resistance.

\footnotetext{
${ }^{1}$ Recebido para publicação em 08/06/98 e na forma revisada em 03/09/98.

${ }^{2}$ Eng $^{\circ}$ Agr $^{\circ}$ Pesquisador, Embrapa Soja. C.P. 231, CEP 86001-970, Londrina/PR.

${ }^{3}$ Estagiário Embrapa Soja, Universidade Estadual de Maringá. C.P. 231, CEP 86001-970, Londrina/PR.

${ }^{4}$ Prof. do Dept ${ }^{\circ}$ de Horticultura, ESALQ/USP. C.P. 9, CEP 13418-900, Piracicaba/SP.

${ }^{5} \mathrm{Eng}^{\circ} \mathrm{Agr}^{\circ}$ do Emater. C.P. 763, CEP 86001-970, Londrina/PR.
} 
Thus, an experiment was carried out at Embrapa Soja, in Londrina, Paraná State, Brazil, in order to 
confirm whether or not there has been resistance of the weed E. heterophylla to the ALS inhibitor herbicides which had been sprayed for several years in a soybean field where the grower has observed failure of the weed control by the ALS inhibitor herbicides. The suspected resistant plants (E-298) from Cafelândia county, Paraná, Brazil, were treated with various doses of several herbicides, compared to a known susceptible biotype. The herbicides and its doses were cloransulan 0,$0 ; 0,015 ; 0,03 ; 0,06 ; 0,12 ; 0,24 \mathrm{~kg}$ a.i./ha, plus Agral $0,2 \% \mathrm{v} / \mathrm{v}$, imazetaphapyr 0,0; 0,$05 ; 0,1 ; 0,2 ; 0,4$ e $0,8 \mathrm{~kg}$ a.i./ha, imazaquin 0,0 ; 0,$075 ; 0,15 ; 0,3 ; 0,6 ; 1,2 \mathrm{~kg}$ a.i./ha, plus Assist $0,3 \% \mathrm{v} / \mathrm{v}$, sulfentrazone 0,$0 ; 0,3 ; 0,6 ; 1,2 ; 2,4$; $4,8 \mathrm{~kg}$ a.i./ha, plus Assist $0,3 \% \mathrm{v} / \mathrm{v}$ and lactofen 0,$0 ; 0,082 ; 0,165 ; 0,33 ; 0,664 ; 1,329 \mathrm{~kg}$ a.i./ha.The

\section{INTRODUÇÃO}

A primeira menção de um possível caso de resistência de plantas daninhas a herbicidas foi observado na agricultura mundial, em 1957, com o produto 2,4-D (Whitehead \& Switzer, 1962). Em 1968, foi relatada a resistência a atrazine (Ryan,1970) e, em 1973, à trifluralina (Mudge et al., 1984). Nos últimos anos, esse assunto tem recebido crescente atenção devido ao aparecimento de espécies resistentes aos herbicidas inibidores da enzima acetolactato sintase (ALS), responsável pela catalase de uma das reações iniciais da via metabólica de produção dos aminoácidos valina, leucina e isoleucina. Existem quatro classes químicas de produtos herbicidas capazes de inibir a ação dessa enzima. Dentre eles, os mais largamente utilizados são os herbicidas pertencentes aos grupos químicos das imidazolinonas, das sulfoniluréias e das triazolopirimidinas (Saari et al., 1994). O uso contínuo desses herbicidas numa mesma área tem levado ao desenvolvimento de biótipos resistentes.

No Brasil, o primeiro caso confirmado de uma população de planta daninha resistente a herbicidas foi relatado por Christoffoleti et al. results indicated different patterns of cross resistance to cloransulan, imazethapyr and imazaquin; and the $\mathrm{GR}_{50}$ ratios were $97.74 ; 11.90$ and 10.86, respectively. The dose response curves of the resistant biotype presented lower values than the susceptible biotype in all the studied rates for the herbicides cloransulan, imazethapyr and imazaquin. On the other hand, there was no difference in the control between the resistant and susceptible biotypes when sulfentrazone and lactofen were sprayed. It was observed efficient control, mainly with the highest rates of both herbicides, proving that there is no multiple herbicide resistance of the wild poinsettia studied.

Key words: cross resistance, multiple resistance, weed, Euphorbia heterophylla.

(1996), com a planta daninha picão-preto (Bidens pilosa) em regiões produtoras de soja.

Vidal \& Fleck (1997) relataram populações de Bidens pilosa, Brachiaria plantaginea e Euphorbia heterophylla resistentes a herbicidas em algumas regiões produtoras de soja no Brasil. Ponchio (1997) também confirmou a existência de populações resistentes de Bidens pilosa a herbicida inibidores da enzima ALS nos Estados do Mato Grosso do Sul e do Rio Grande do Sul. Gazziero et al.(1997) confirmaram a resistência de um biótipo da planta daninha Brachiaria plantaginea aos herbicidas inibidores da enzima ACCase, provenientes de uma área de cultura de soja da região sudoeste do Estado do Paraná. Christoffoleti et al. (1998), também confirmaram a resistência de populações de capim marmelada no Estado do Paraná.

As plantas resistentes surgem, principalmente, em áreas de monocultura onde aplicações repetidas de um único e efetivo herbicida se estendem anos a fio, sem modificações, causando mudanças na flora infestante (Maxwell et al., 1990; Blair, 1991). E certos indivíduos (biótipos) dentro da população são capazes de sobreviver a aplicações desse produto mais do que outros. Uma revisão sobre os 
principais avanços e descobertas na área de plantas daninhas resistentes aos herbicidas foi feita por Christoffoleti et al. (1994).

A utilização de herbicidas com o mesmo mecanismo de ação, pode possibilitar o desenvolvimento de biótipos resistentes. Mesmo quando agricultores alternam herbicidas e classes de herbicidas, seguindo orientação técnica, podem estar utilizando classes diferentes mas com o mesmo mecanismo de ação. Essa condição cria o potencial para o desenvolvimento de infestantes resistentes (Powles \& Preston, 1995).

Outro fato importante também pode ocorrer em regiões, onde as condições ambientais não são favoráveis à decomposição do herbicida e à maior persistência do produto no solo, favorecerá o processo de seleção de populações de plantas daninhas resistentes, sendo maior a pressão de seleção exercida sobre a população de plantas daninhas, principalmente se houver múltiplos fluxos de emergência de sementes no mesmo ano agrícola (Weller, 1992; Hartzler \& Owen, 1993).

Recentemente, têm sido observados diversos campos de produção de soja no Brasil com falhas de controle do amendoim-bravo pelos herbicidas inibidores da enzima ALS recomendados para essa cultura. Nessa situação, tem-se atribuído como causas dessas falhas o desenvolvimento de biótipos resistentes sem nenhuma comprovação científica.

Sendo assim, este experimento foi conduzido com o objetivo de confirmar cientificamente a resistência de um biótipo (E298) de amendoim-bravo aos herbicidas inibidores da ALS e observar o nível de resistência cruzada entre herbicidas inibidores da enzima ALS pertencentes a diferentes grupos químicos, bem como, verificar se existe resistência múltipla com herbicidas de outros mecanismos de ação.

\section{MATERIAL E MÉTODOS}

$\mathrm{O}$ experimento foi conduzido em área pertencente à Embrapa Soja, Londrina, PR, durante o período de 4 de setembro a 20 de outubro de 1997.

Sementes de amendoim-bravo foram coletadas na localidade Central dos Jesuítas, município de Cafelândia, PR, onde a cultura da soja vinha sendo cultivada por vários anos com aplicações sucessivas de herbicidas inibidores da enzima ALS (imazaquin principalmente). Na safra 95/96, foi observado que a aplicação do herbicida imazethapyr estava apresentando falhas de controle dessa planta daninha. Paralelamente, também foram colhidas sementes em áreas onde não havia sido utilizado nenhum tipo de herbicida, considerando-se essas plantas como suscetíveis aos herbicidas inibidores da enzima ALS.

$\mathrm{O}$ experimento foi conduzido em delineamento experimental em blocos casualizados com quatro repetições. Os tratamentos foram estabelecidos considerando-se as doses recomendadas dos herbicidas, metade delas, e duas, quatro e oito vezes superiores a recomendada. Os produtos e as doses aplicadas foram cloransulan nas doses 0,$0 ; 0,015 ; 0,03$; 0,$06 ; 0,12 ; 0,24 \mathrm{~kg}$ i.a./ha, mais o espalhante adesivo Agral 0,2\% v/v, imazetaphapyr nas doses 0,$0 ; 0,05 ; 0,1 ; 0,2 ; 0,4$ e $0,8 \mathrm{~kg}$ i.a./ha, imazaquin nas doses 0,$0 ; 0,075 ; 0,15 ; 0,3 ; 0,6 ; 1,2 \mathrm{~kg}$ i.a./ha, mais o espalhante adesivo Assist $0,3 \% \mathrm{v} / \mathrm{v}$, sulfentrazone nas doses: 0,$0 ; 0,3 ; 0,6 ; 1,2 ; 2,4$; $4,8 \mathrm{~kg}$ i.a./ha, mais o espalhante adesivo Assist $0,3 \% \mathrm{v} / \mathrm{v}$, e lactofen nas doses 0,$0 ; 0,082 ; 0,165$; 0,$33 ; 0,664 ; 1,329 \mathrm{~kg}$ i.a./ha.

Sementes de amendoim-bravo provenientes do biótipo considerado suscetível e daquele onde suspeitava-se da resistência foram semeadas separadamente em copos plásticos de $500 \mathrm{ml}$ de capacidade, no mesmo dia. O material utilizado para o enchimento dos copos plásticos foi latossolo roxo eutrófico.O experimento foi mantido em condições de campo, em área próxima à casa-de-vegetação, com o intuito de proporcionar condições as mais próximas possíveis daquelas que originalmente possuem as plantas de amendoim-bravo. Os copos foram irrigados regularmente, mantendo-se a umidade do solo próxima à capacidade de campo. Após a 
germinação e a emergência, as plantas foram desbastadas, deixando-se três plantas por copo. 
Todos os produtos foram aplicados no dia 29 de setembro, em condições de pós-emergência, quando o amendoim-bravo apresentava-se com quatro a cinco folhas $(4,6 \mathrm{~cm})$, para o biótipo resistente, e cinco a seis folhas $(6,2 \mathrm{~cm})$, para o suscetível. Os herbicidas foram aplicados por pulverizador costal pressurizado a $\mathrm{CO}_{2}$ com pressão de $40 \mathrm{lb} / \mathrm{pol}^{2}$, contendo barra de pulverização de quatro bicos 110015 e volume de pulverização de 1601/ha. A temperatura do ar durante a aplicação era de $24{ }^{\circ} \mathrm{C}$, com umidade relativa de $66 \%$.

As avaliações de controle foram feitas aos sete dias, após a aplicação dos herbicidas sulfentrazone e lactofen, e aos 14 dias para cloransulan, imazethapyr e imazaquin, através da escala visual de $0-100 \%$, onde $0 \%$ (zero) representava nenhum controle e $100 \%$ o controle total. Foram ajustados modelos de regressão (potência e exponencial) aos dados observados, tendo como variável resposta a percentagem de controle de plantas daninhas e a variável explanatória doses de herbicidas. Os valores de $\mathrm{GR}_{50}$ (dose necessária para proporcionar $50 \%$ de controle do biótipo resistente e do suscetível) foram obtidos a partir dos modelos ajustados. E, em seguida, calculadas as relações médias de $\mathrm{GR}_{50}$ para cada produto, dividindo-se o $\mathrm{GR}_{50}$ do biótipo resistente pelo do biótipo suscetível (R/S). O valor encontrado expressa o número de vezes em que a dose necessária para controlar $50 \%$ das plantas do biótipo suscetível deve ser superior para que possa ocorrer o mesmo efeito com o biótipo resistente.

Todo material vegetal e sementes restantes dos testes foram eliminados para evitar alguma disseminação involuntária do biótipo resistente.

\section{RESULTADOS E DISCUSSÃO}

Embora os biótipos, resistente (E-298) e suscetível, tenham sido semeados no mesmo dia, verificou-se crescimento mais rápido do suscetível, o que levou à aplicação dos produtos em plantas com tamanhos diferentes. Assim, as plantas do biótipo suscetível estavam mais desenvolvidas no momento da aplicação, o que pode, em parte, ter reduzido a eficiência do herbicida.

Destaca-se, pela Tabela 1, que os controles obtidos para o biótipo suscetível, nas doses recomendadas, foram abaixo dos esperados nos resultados de campo. Este fato deve-se, provavelmente, às condições edáficas e climáticas, principalmente, água no solo, serem muito diferenciadas em copos plásticos com capacidade de $500 \mathrm{ml}$ quando comparadas ao solo em condições de campo.

TABELA 1. Percentagem média de controle de amendoim-bravo (Euphorbia heterophylla), biótipo resistente E-298 (R) e suscetível (S), em função das doses de herbicidas. Embrapa Soja, Londrina, PR, 1998.

\begin{tabular}{lcccccccccc}
\hline Doses $^{1}$ & \multicolumn{2}{c}{ cloransulan } & \multicolumn{2}{c}{ imazethapyr } & \multicolumn{2}{c}{ imazaquin } & \multicolumn{2}{c}{ sulfentrazone } & \multicolumn{2}{c}{ lactofen } \\
\hline & $\mathrm{R}$ & $\mathrm{S}$ & $\mathrm{R}$ & $\mathrm{S}$ & $\mathrm{R}$ & $\mathrm{S}$ & $\mathrm{R}$ & $\mathrm{S}$ & $\mathrm{R}$ & $\mathrm{S}$ \\
\hline $0,5 \mathrm{X}$ & 13,8 & 42,5 & 0,0 & 45,0 & 6,3 & 42,5 & 98,8 & 93,8 & 86,3 & 55,0 \\
$1,0 \mathrm{X}$ & 18,8 & 50,0 & 1,3 & 57,5 & 10,0 & 52,5 & 100,0 & 95,8 & 93,3 & 66,3 \\
$2,0 \mathrm{X}$ & 18,8 & 58,8 & 1,3 & 65,0 & 13,8 & 53,8 & 100,0 & 98,0 & 98,8 & 83,8 \\
$4,0 \mathrm{X}$ & 21,3 & 56,3 & 10,0 & 75,0 & 17,5 & 58,3 & 100,0 & 100,0 & 99,0 & 93,8 \\
$8,0 \mathrm{X}$ & 28,8 & 61,3 & 36,3 & 82,5 & 25,0 & 65,0 & 100,0 & 100,0 & 100,0 & 98,8 \\
\hline
\end{tabular}

1- as doses estão representadas pelo número de vezes em relação as doses recomendadas.

Foi obtida uma percentagem média de controle do amendoim-bravo de $28,8 \%$ (biótipo E-298) e 61,3\% (biótipo suscetível), quando se utilizou o herbicida cloransulan na maior dose (Tabela 1). As doses que proporcionaram $50 \%$ de redução no crescimento das plantas foram obtidas 
por estimativa da equação ajustada, assim sendo, alguns valores encontram-se fora da região das doses testadas. Os valores de $\mathrm{GR}_{50}$ para este produto, obtidos a partir do modelo ajustado, 
foram > 0,24 e 0,035 kgi.a./ha, para o biótipo resistente (E-298) e o suscetível, respectivamente (Figura 1). Isso significa que o biótipo E-298 necessita de uma dose superior a 6,8 vezes, em relação à aplicada na população suscetível, para causar uma redução de $50 \%$ do seu crescimento
(Tabela 2). Kissman (1995) relatou que biótipos podem apresentar níveis diversos de suscetibilidade ou resistência a determinado herbicida. E, em casos extremos, foram constatadas resistência a doses de até 100 vezes a dose que normalmente controla biótipos suscetíveis da mesma espécie.

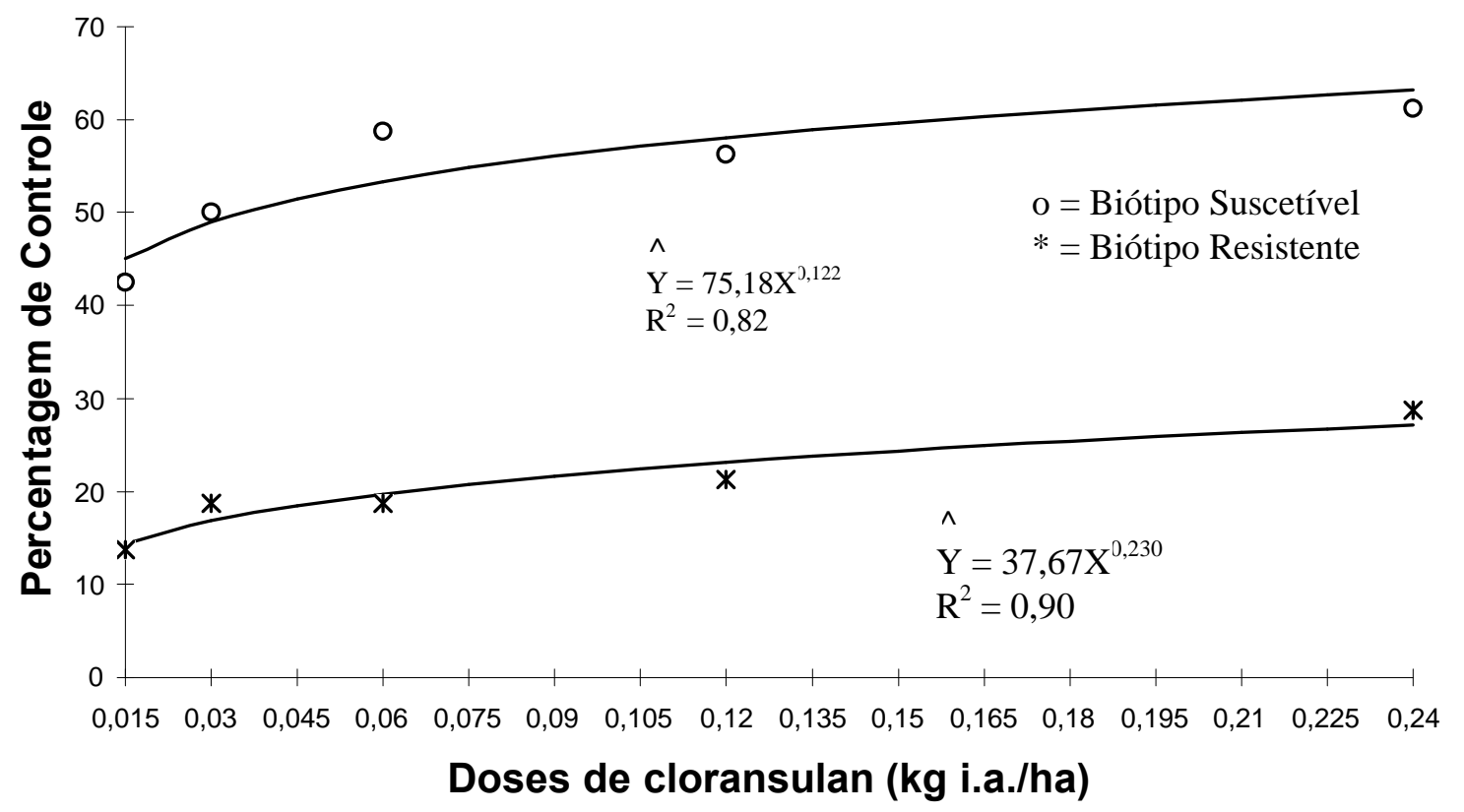

FIGURA 1. Percentagem de controle do amendoim-bravo em função das doses do herbicida cloransulan. Embrapa Soja, PR, 1998.

TABELA 2. Doses ( $\mathrm{kg}$ i.a./ha) correspondentes aos $\mathrm{GR}_{50}$ do biótipo resistente (R) e do biótipo suscetível (S) e a relação R/S. Embrapa Soja, Londrina,PR, 1998.

\begin{tabular}{|c|c|c|c|}
\hline \multirow[t]{2}{*}{ Herbicidas } & \multicolumn{2}{|c|}{$\mathrm{GR}_{50}$} & \multirow[t]{2}{*}{$\mathrm{R} / \mathrm{S}$} \\
\hline & Resistente (R) & Suscetível (S) & \\
\hline cloransulan & $>0,24$ & 0,035 & $>6,85$ \\
\hline imazethapyr & 0,762 & 0,064 & 11,90 \\
\hline imazaquin & $>1,2$ & 0,167 & $>7,18$ \\
\hline
\end{tabular}

Quando foi aplicado o imazethapyr na maior dose, a percentagem média de controle para o biótipo E-298 foi baixa (36,3\%), enquanto o suscetível alcançou 82,5\% (Tabela 1). A dose de herbicida que proporcionou $50 \%$ de mortalidade das plantas do biótipo E-298 foi 0,762 kg i.a./ha e, para o suscetível, este valor foi $0,064 \mathrm{~kg}$ i.a./ha
(Figura 2). Vidal et al. (1997 b) verificaram que, de um total de 472 plantas de amendoim-bravo tratadas com imazethapyr, $40 \%$ delas mostraramse resistentes ao produto em experimentos realizados na região de Passo Fundo, RS. 
A aplicação de oito vezes a dose recomendada do herbicida imazaquin proporcionou 
percentagens médias de controle do amendoimbravo equivalentes a $25 \%$ e $65 \%$, para os biótipos E-298 e suscetível, respectivamente (Tabela 1).
Os valores de $\mathrm{GR}_{50}$ obtidos através dos modelos ajustados foram $>1,2 \mathrm{~kg}$ i.a./ha (biótipo E-298) e 0,167 kg i.a./ha (biótipo suscetível) (Figura 3).

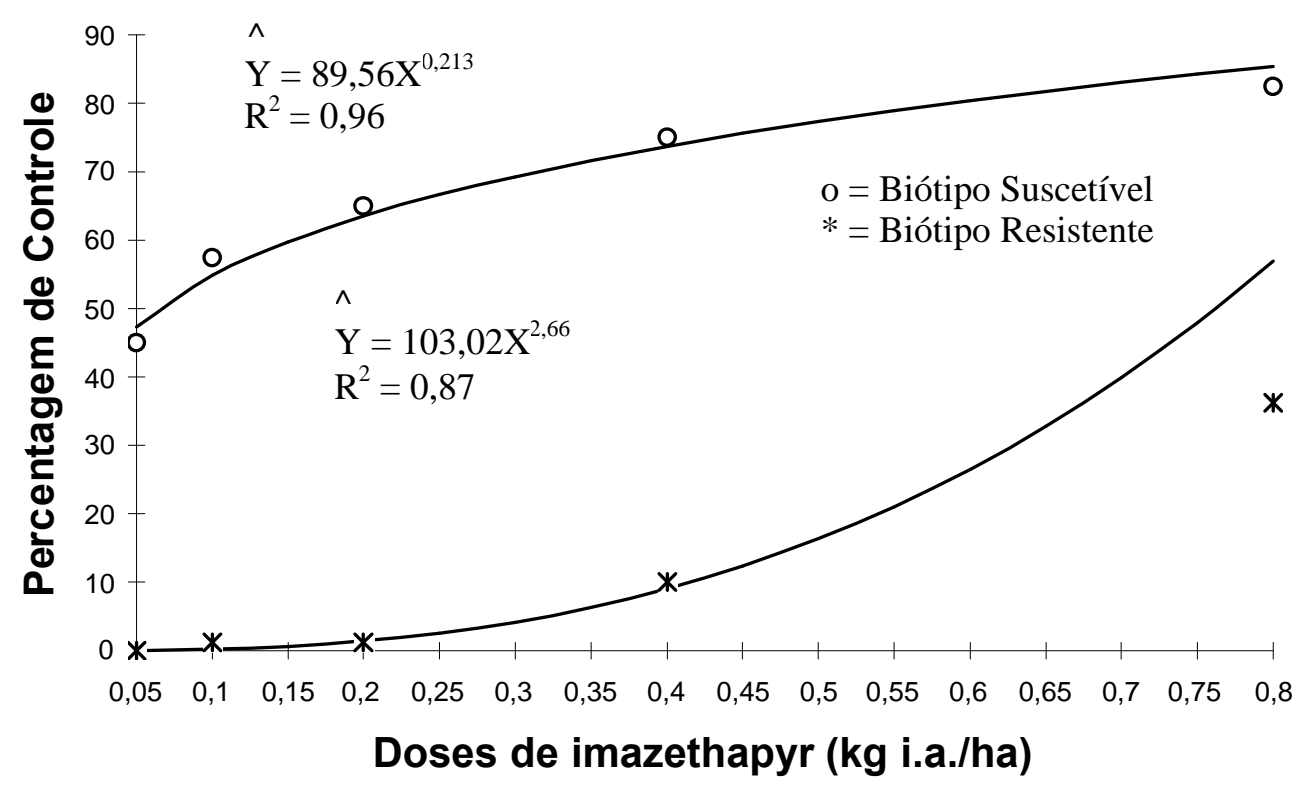

FIGURA 2. Percentagem de controle do amendoim-bravo em função das doses do herbicida imazethapyr. Embrapa Soja, PR, 1998.

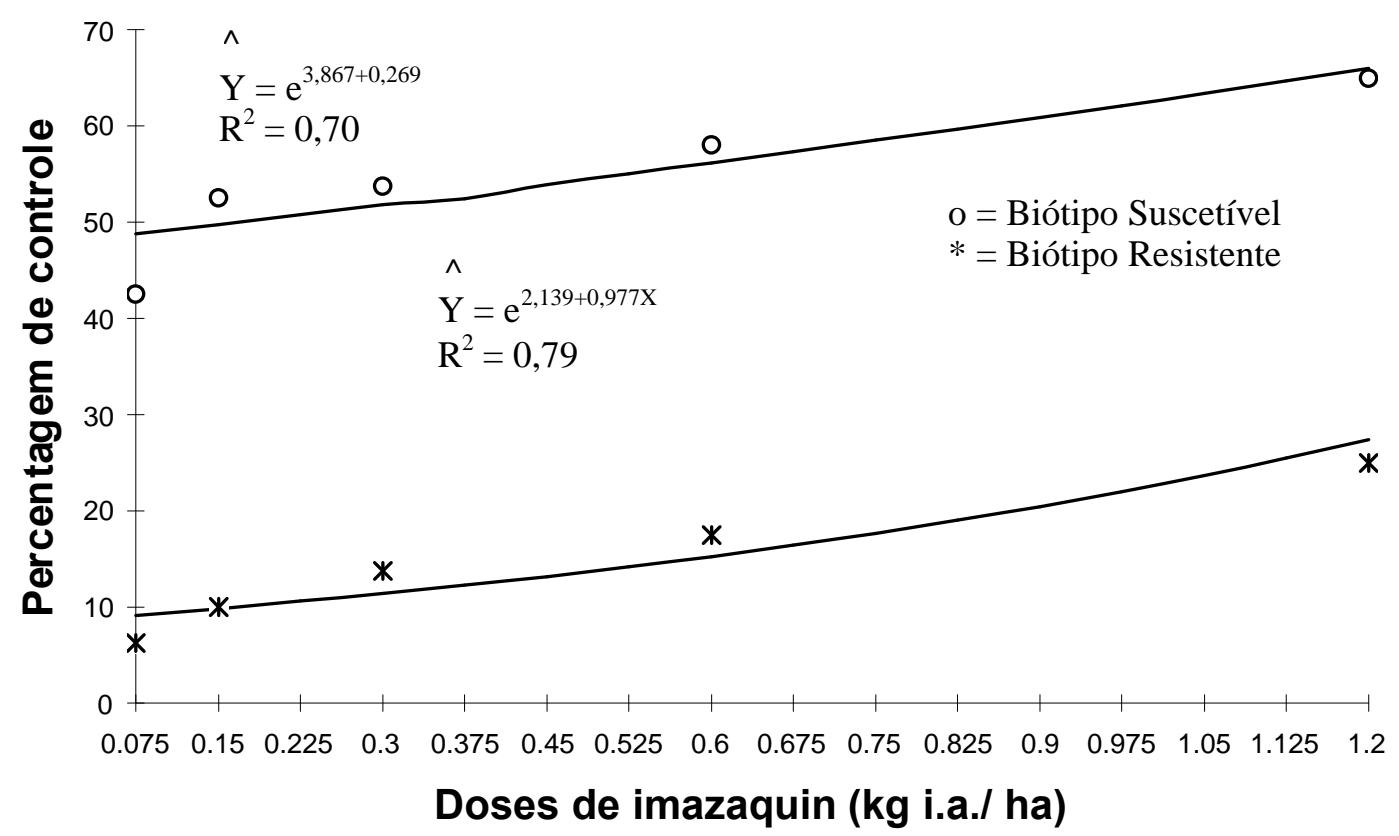

FIGURA 3. Percentagem de controle do amendoim-bravo em função das doses do herbicida imazaquin. Embrapa Soja, Londrina, PR, 1998. 
O herbicida sulfentrazone, nas cinco doses aplicadas, apresentou controle acima de 93\%, tanto para o biótipo E-298, quanto para o suscetível (Tabela 1). Não foi possível o ajuste de modelos matemáticos para explicar os dados de percentagem média de controle do amendoimbravo, em função das doses do sulfentrazone para o biótipo E-298, devido ao alto controle com a aplicação de todos os tratamentos. Assim sendo, foram plotados no gráfico apenas os pontos observados (Figura 4). Os resultados indicaram que os biótipos de amendoim-bravo avaliados não apresentaram resistência ao sulfentrazone.

O herbicida lactofen também mostrou- eficiente no controle do amendoim-bravo, resultando em percentagem média de controle $100 \%$ (biótipo E-298) e 98,9\% (biótipo suscetível), na maior dose (Tabela 1). Da mesma maneira que o sulfentrazone, os biótipos de amendoim-bravo avaliados também não apresentaram resistência ao lactofen, não sendo possível determinar os valores de $\mathrm{GR}_{50}$. Isso ocorreu em função da menor dose aplicada ser suficiente para proporcionar um controle acima de 50\% (Figura 5). Vidal et al. (1997 a), trabalhando com amendoim-bravo (biótipo ER-8) resistente aos herbicidas inibidores da ALS, verificaram que o lactofen apresentou controle satisfatório do biótipo testado.

se

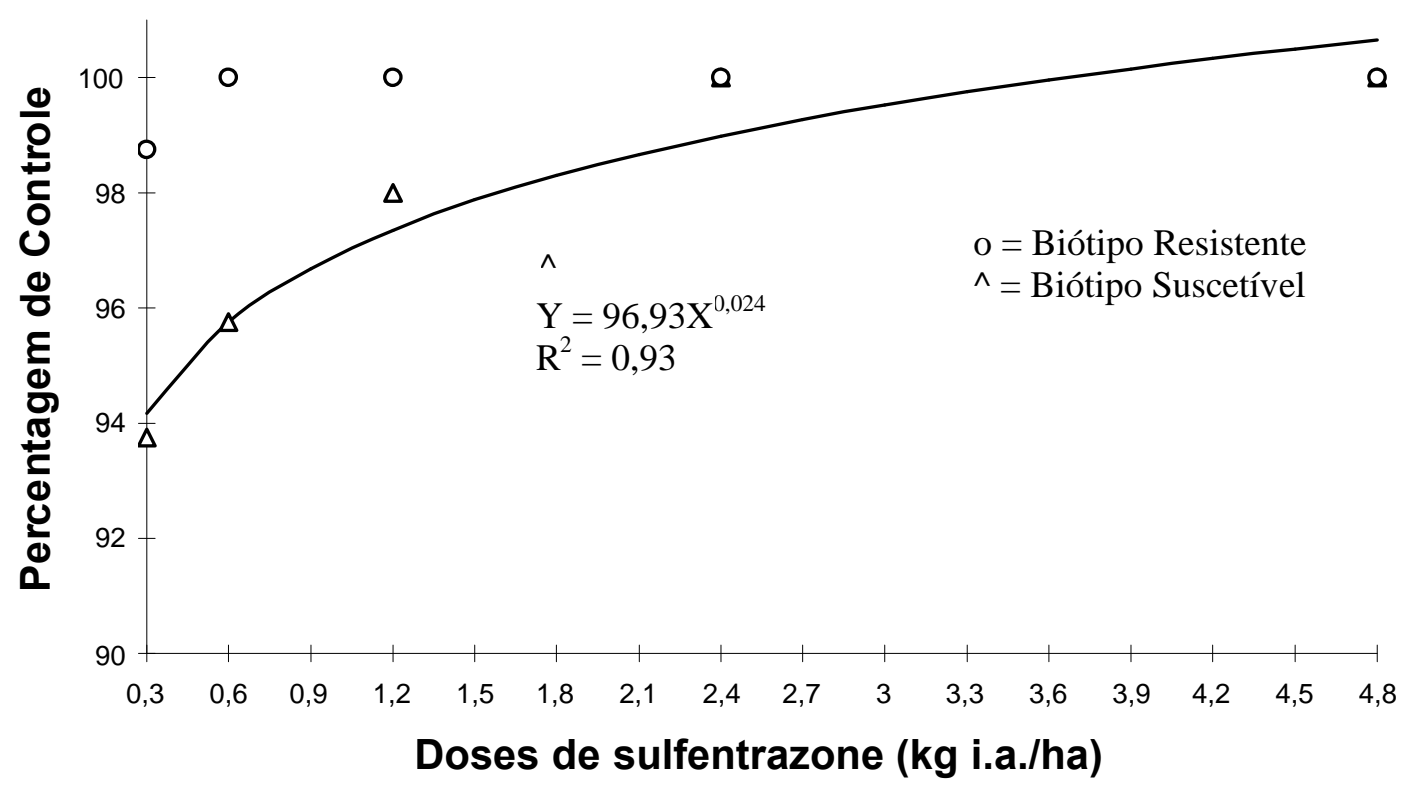

FIGURA 4. Percentagem de controle do amendoim-bravo em função das doses do herbicida sulfentrazone. Embrapa Soja, Londrina, PR, 1998.

As relações médias de $\mathrm{GR}_{50}$ resistente/GR 50 suscetível (R/S) obtidas foram > 6,$85 ; 11,90$ e $>7,18$, para os herbicidas cloransulan, imazethapyr e imazaquin, respectivamente (Tabela 2). Isso significa que a população do biótipo E-298 sofreu redução de $50 \%$ no crescimento, a partir de doses de cloransulan, imazethapyr e imazaquin muito superiores em relação às aplicadas na população suscetível, podendo-se dizer que o biótipo E-298 apresentou diferentes níveis de resistência cruzada a esses produtos.

Para os herbicidas sulfentrazone e lactofen, houve controle satisfatório do amendoim-bravo biótipo E-298 e suscetível. Esses resultados indicam que o mecanismo de resistência do biótipo de amendoim-bravo, 
provavelmente, não é de resistência múltipla aos herbicidas com diferentes mecanismos de ação. 


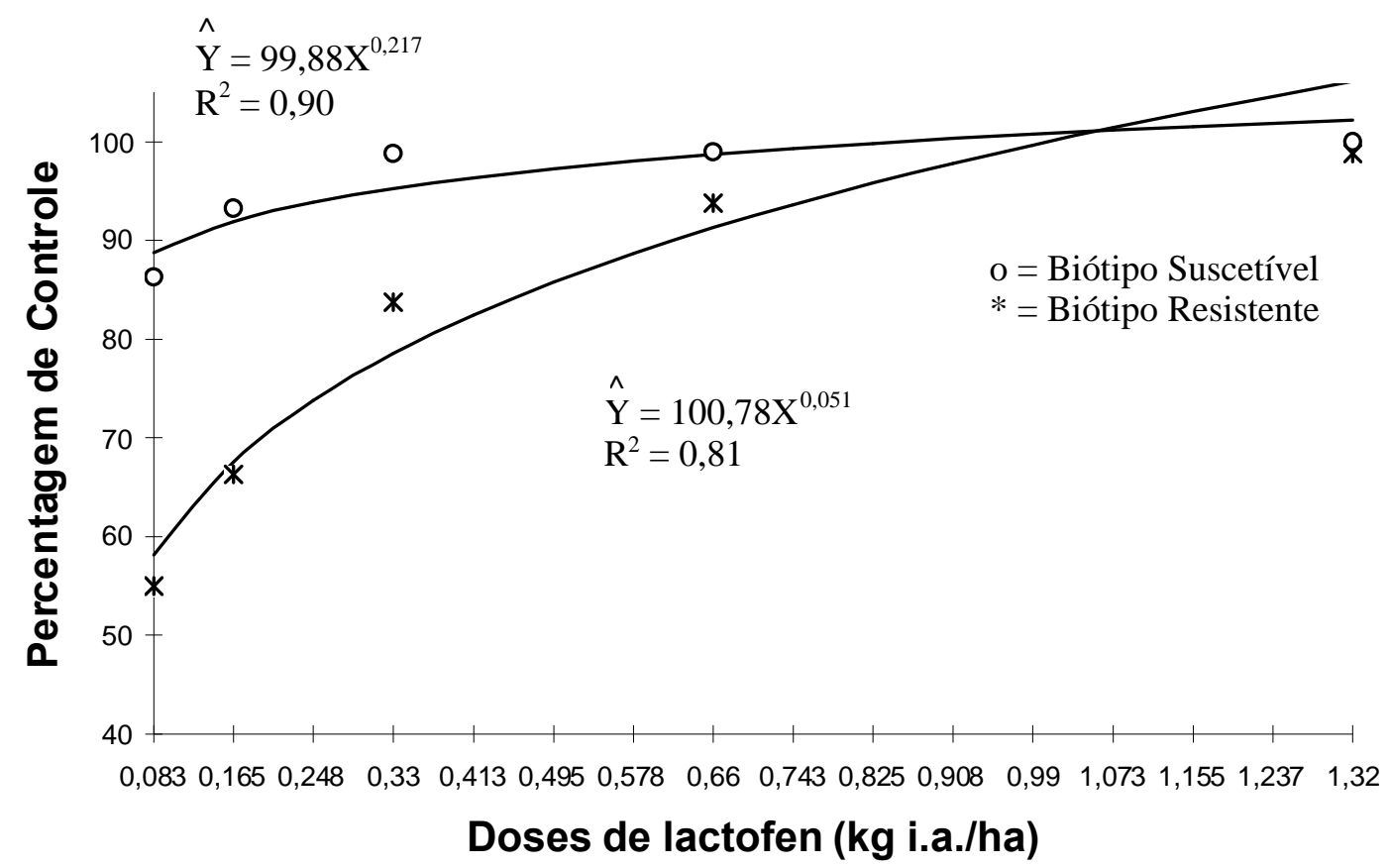

FIGURA 5. Percentagem de controle do amendoim-bravo em função das doses do herbicida lactofen. Embrapa Soja, Londrina, PR, 1998.

Uma das estratégias recomendadas para 0 controle de plantas daninhas resistentes é a combinação de herbicidas de diferentes mecanismos de ação (Vidal et al. 1997 b). Deste modo, o sulfentrazone e o lactofen podem se constituir em alternativas para o controle de amendoim-bravo em áreas com a presença do biótipo resistente aos inibidores da enzima ALS.

\section{AGRADECIMENTOS}

Os autores agradecem aos colegas pesquisadores/estatísticos $\operatorname{Dr}^{\mathrm{a}}$ Maria Cristina Neves de Oliveira, Dr. José Erivaldo Pereira da Embrapa Soja, aos técnicos agrícolas da Embrapa Soja, Mário Nakano e a Reinaldo Teruhico Moriyama pela colaboração.

\section{LITERATURA CITADA}

BLAIR, W. Herbicide resistance in weeds: an overview. American Cyanamid, Agricultural Division, Crop Protection Chemicals Departament. Princeton, New Jersey; 16 p.,1991.
CHRISTOFFOLETI, P.J., CORTEZ, M.G., VICTÓRIA FILHO, R. Resistance of alexanderweed (Brachiaria plantaginea) to ACCase inhibitor herbicides in soybean from Paraná State - Brazil.1998. MEETING OF THE WEED SCIENCE SOCIETY OF AMERICA. Chicago. WSSA Abstract. p.65, 1998.

CHRISTOFFOLETI, P.J., PONCHIO, J.A.R., BERG, E.V.D., VICTÓRIA FILHO, R. Imidazolinone resistant Bidens pilosa byotipes in the Brazilian soybeans areas. 1996. MEETING OF THE WEED SCIENCE SOCIETY OF AMERICA. Norfolk. WSSA Abstract. p.10, 1996.

CHRISTOFFOLETI, P.J., VICTÓRIA FILHO, R., SILVA, C.B. Resistência de plantas daninhas aos herbicidas. Planta Daninha, v.12, n.1, p.13-20, 1994.

GAZZIERO, D.L.P., CHRISTOFFOLETI, P.J., MACIEL, C.D.M., SCARAMUZZA Jr., J.R. Resistência de biótipos de Brachiaria 
plantaginea aos herbicidas inibidores da ACCase aplicados em soja. In: CONGRESSO BRASILEIRO DA CIÊNCIA DAS PLANTAS DANINHAS, 21, 1997, Caxambú. Resumos... Caxambú: SBCPD, 1997. p.88.

HARTZLER, R.G., OWEN, M.D.K. Herbicide resistance - concerns and management. Integrated pest management 39. Iowa State University, Ames, I.A. 8 p., 1993.

KISSMANN, K.G. Resistência de plantas daninhas a herbicidas. BASF, 32 p.1995.

MAXWELL, B.D., ROUSH, M.L. e RADOSEVICH, S.R. Predicting the evolution and dynamics of herbicide resistance in weed populations. Weed Tech., v. 4, p.2-13, 1990.

MUDGE, L.C., GOSSETT, B.J., MURPHY, T.R. Resistance of goosegrass (Eleusine indica) to dinitroaniline herbicides.Weed Sci.,v.32,p.591-594, 1984.

PONCHIO, J.A.R. Resistência de Bidens pilosa L. aos herbicidas inibidores da enzima acetolactato sintase. ESALQ-Piracicaba, 1997. 120p.(Tese Doutorado).

POWLES, S.B., PRESTON, C. Herbicide cross resistance and multiple resistance in plants. Departament of crop protection. Waite Agricultural Research Institute, University of Adelaide. South Australia, 34 p., 1995.

RYAN, G.F. Resistance of common groundsel to simazine and atrazine. Weed Sci.: v.18, p.614-616, 1970.

SAARI, L.L., COTTERMAN, J.C., THILL, D.C.
Resistence to acetolactato synthase inhibiting herbicides. In: POWLES, S. B. e HOLTUM, J. A. M. Herbicide Resistance in Plants: Biology and Biochemistry. Eds Lewis Publishers, Boca Raton, FL, p.83139, 1994.

VIDAL, R.A., FLECK, N.G., THEISEN, G., NEVES, R., PETRY, L.A. Picão-preto e leiteira resistentes aos inibidores da ALS não apresentam resistência aos herbicidas com diferentes mecanismos de ação. CONGRESSO BRASILEIRO DA CIÊNCIA DAS PLANTAS DANINHAS, 21, 1997, Caxambú. Resumos... Caxambú: SBCPD, 1997a. p.465.

VIDAL, R.A., OLIVEIRA, N.A., FLECK, N.G., GUIMARÃES, F.B., SILVA, N.G. Misturas de mecanismos de ação de herbicidas no controle de leiteira resistente aos inibidores da ALS. CONGRESSO BRASILEIRO DA CIÊNCIA DAS PLANTAS DANINHAS, 21, 1997, Caxambú. Resumos... Caxambú: SBCPD, 1997b. p.468.

VIDAL, R.A., FLECK, N.G. Three weed species with confirmed resistance to herbicides in Brazil.1997. MEETING OF THE WEED SCIENCE SOCIETY OF AMERICA. WSSA Abstract. p.100, 1997.

WELLER, S.C. Herbicide resistance in weeds. In: Herbicide action: an intensive course on activity, selectivity, behavior and fate of herbicides in plants and soils. Purdue University. West Lafayette, I.N. 666 p., 1992.

WHITEHEAD, C.W. \& SWITZER, C.M. The differencial response of strains of wild carrot to 2,4-D and related herbicides. Can. J. Plant Sci., v.43, p.255-262, 1962. 